Article

\title{
Structural Design and Experimental Analysis of a Piezoelectric Vibration Feeder with a Magnetic Spring
}

\author{
Xiaochao Tian, Zhigang Yang, Yong Liu *, Yanhu Shen and Song Chen \\ Institute of Mechanical Science and Engineering, Jilin University, Changchun 130022, China; \\ E-Mails: tianxczb@163.com (T.X.); yzg@jlu.edu.cn (Y.Z.); shenyanhu0525@163.com (S.Y.); \\ zhibangcs@sina.cn (C.S.)
}

* Author to whom correspondence should be addressed; E-Mail: yongliu@jlu.edu.cn; Tel.: +86-519-81584393; Fax: +86-519-81584395.

Received: 25 June 2014; in revised form: 2 August 2014 / Accepted: 5 August 2014 /

Published: 19 August 2014

\begin{abstract}
A piezoelectric vibration feeder with a magnetic spring is discussed in this paper. The feeder can keep resonance frequency relatively stable under changing loading. Through the analysis on the working principle and magnetic spring stiffness characteristic of this feeder, the dynamic model was established and the relationship among system resonance frequency, loading and magnetic spring stiffness was obtained. The analysis showed that, as the loading changed, the magnetic spring stiffness changed accordingly, which maintained a trend of stability in the system resonance frequency. A prototype was made for the experiment, and the relationship among the loading, magnetic spring axial clearance and system resonance frequency was obtained. The result showed that, when the loading changes, the resonance frequency and feeding speed tended to be stable, which matched the theoretical analysis. Through comparison with a traditional vibration feeder, within nominal loading, this new feeder has more stable resonance frequency and feeding speed.
\end{abstract}

Key words: piezoelectric vibration feeder; resonance; magnetic spring; axial clearance

\section{Introduction}

Because of good patterning, guiding performance, and high efficiency, a vibrating feeder is widely used in automatic assembly, processing and inspection, etc. [1]. The traditional vibrating feeder takes mainly mechanical or electromagnetic drives, but there are disadvantages, like high noise level, low 
feeding accuracy, high power consumption, and easily interfered by electromagnetism, and so on [2]. With the advanced industry development, the demand on vibrating feeder is increasing greatly. In the end of 1970s, Japan invented rectangular piezoelectric bimorph as the driving source for cantilever piezoelectric vibrating feeder [3]. There have been more invention patents and related research on different structure feeders since then [4-6]. The spiral piezoelectric vibrating hopper was invented in the USA. It had adverse phase AC voltage and the piezoelectric film drove the spring leaf vibration back and forth [7]. The agile manufacturing and system lab in Inha University South Korea invented a new piezoelectric feeder. Finite Element Analysis (FEA) and experiment analysis were done on the feeder [8]. Taiwanese researchers analyzed the linear piezoelectric vibration feeder. The Rayleigh-Ritz method, model and experiment were carried out to ascertain the factors that influence system feeding speed [9]. Nowadays, in China, some universities are working on piezoelectric vibrating feeder research [10-18], such as Tianjin University, Dalian Science and Technology University, Nanjing University of Aeronautics and Astronautics, Jilin University, etc.

To increase efficiency, feeders are usually working at resonance conditions. During feeding, once the materials are put on the feeder, with the feeding, the material mass reduces gradually, since the present feeder has fixed spring stiffness, which cannot change along with the material mass (loading). Therefore, the feeder resonance frequency increases, and the drive output frequency needs to be constantly increased to make the feeder work at resonance conditions. Moreover, when adding materials later, the drive output frequency needs to be reduced back again. Nowadays, the drive with frequency tracking and auto-controlling function is expensive, and there is a lag during working. If the loading changes, the feeder spring stiffness can change accordingly, and then the resonance frequency can function as well. At small deformation, the magnetic spring made of two annular magnets has stable stiffness, and when axial clearance is reduced, the stiffness increases. In addition, with the same structure and size, the annular magnetic spring has good mechanical properties and wide changing range [19,20], which can simplify the system, reduce the noise level, and can eliminate fatigue deformation.

Based on adjustable stiffness of the magnetic spring, a novel piezoelectric resonance feeder is proposed. With theoretical analysis and experiment, the material mass, magnetic spring stiffness and system resonance frequency changes were analyzed initially, and the possibility to stabilize the system resonance frequency with the magnetic spring was validated.

\section{Structure and Working Concept}

The structure of the magnetic spring piezoelectric vibration feeder is shown in Figure 1 below. The driving source is the piezoelectric bimorph vibrator, which is fixed with the top plate by the mass block. The vibration bar is connected with the centers of the piezoelectric vibrator and suspended magnet, and fixed with nuts from both sides. There is a center-facing in homopolarity between the suspended magnet and permanent magnet. The permanent magnet is mounted in the base, there are rubber feet below the base to reduce vibration, and the base and top plate are connected with a supporting at $75^{\circ}$. The top plate, mass block, connecting ring, piezoelectric vibrator, suspended magnet and vibration bar can be treated in their entirety as a vibration amplified system, while the suspended magnet and permanent magnet with homopolar that face each other can be used as a compressed magnetic spring. 
Figure 1. Structure of Magnetic spring piezoelectric vibration feeder.

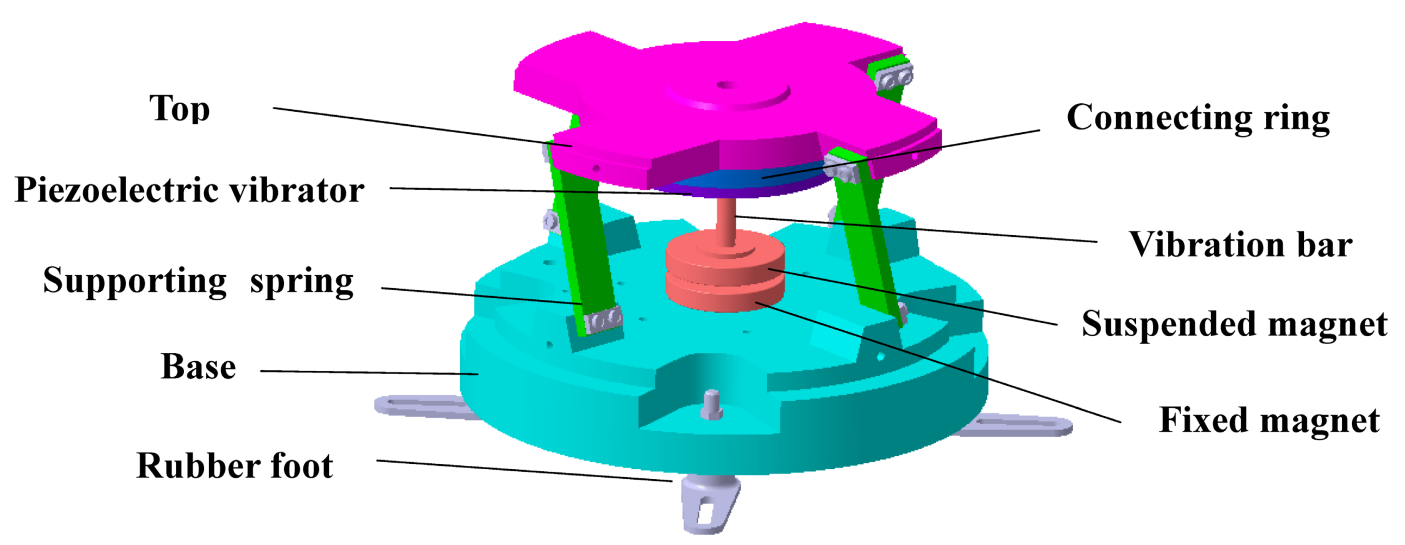

In magnetic spring piezoelectric vibration feeder, sine $\mathrm{AC}$ voltage is placed on piezoelectric bimorph vibrator to obtain bending deformation. When the voltage changes, the output displacement of the piezoelectric vibrator changes accordingly, and longitudinal vibration happens, and this vibration forces the supporting springs with the same inclination to create and release the deformation. In this case, longitudinal vibration and torsional movement are combined, and the magnetic spring amplifies the system vibration. In this situation, the material in the tray moves in sequence, and the feeding works.

\section{Stiffness Characteristic and Dynamic Model}

\subsection{Magnetic Spring Stiffness Characteristic}

Within small displacement of the magnetic spring, the relationship between displacement and loading is close to linear. As shown in Figure 2, take small clearance of the magnetic spring as $x_{1}, x_{2}$, the middle of $x_{1}$ and $x_{2}$ as $x_{0}$, corresponding value as $f\left(x_{0}\right), f\left(x_{0}\right)^{\prime}$ as the slope, which can be used as the magnetic spring stiffness $k_{0}$, within small distance. Increase the distance, marked as $x_{3}$, take the middle of $x_{1}$ and $x_{3}$ as $x_{0}$ ', corresponding value $f\left(x_{0}{ }^{\prime}\right), f\left(x_{0}{ }^{\prime}\right)^{\prime}$, as the stiffness $k_{0}$ ', between $x_{1}$ and $x_{3}$. From the figure, $\left|f\left(x_{0}\right)^{\prime}\right|>\left|f\left(x_{0}{ }^{\prime}\right)^{\prime}\right|$, which means $k_{0}>k_{0}$ ', shows that, with an increase in distance, the axial stiffness reduces, and vice versa.

Figure 2. Relationship between loading and axial clearance.

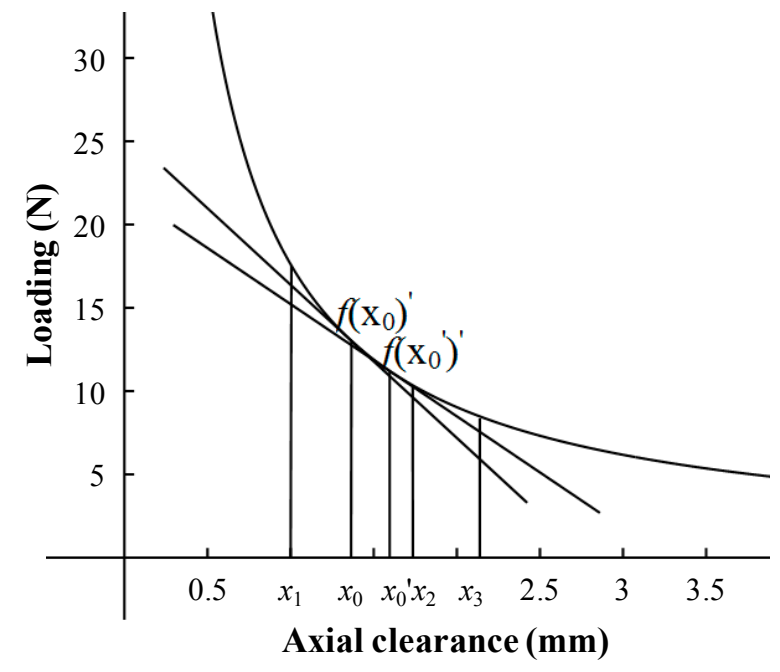




\subsection{Dynamic Formula}

The mechanical model of the magnetic spring piezoelectric vibration feeder is shown in Figure 3. $M$ is the equivalent mass of the entire system, including top plate, mass block, connecting ring, vibration bar. $k_{0}$ is the equivalent stiffness of the piezoelectric vibrator, $k_{1}$ is the stiffness of the magnetic spring. From Section 3.1, the magnetic spring shows linear stiffness within a small distance. Therefore, it can be used for linear calculation. $k_{2}$ is the stiffness of the supporting spring every $90^{\circ}$. The rubber feet act to reduce the vibration of the spring. If the stiffness is low it can be ignored.

$y_{0}$ is the vibration displacement of the piezoelectric vibrator. Supposing $y_{0}=A \cos (\omega t), y$ is the vibration displacement of the top plate, $F_{0}$ is the initial excitation, $F_{0}=k_{0} y_{0}$ and $c$ is the system equivalent damping. The single degree of freedom with damping forced vibration system has a differential equation of motion

$$
\left\{\begin{array}{l}
M \ddot{y}+c \dot{y}+k_{0}\left(y-y_{0}\right)+k_{2} y=0 \\
F_{0}+k_{0}\left(y-y_{0}\right)-k_{1} y_{0}=0
\end{array}\right\}
$$

which can be transferred as

$$
M \ddot{y}+\mathrm{c} \dot{y}+\frac{k_{1} k_{2}+k_{1} k_{0}+k_{2} k_{0}}{k_{1}+k_{0}} y=\frac{k_{0}}{k_{1}+k_{0}} F_{0}
$$

Furthermore,

$$
\ddot{y}+\frac{c}{M} \dot{y}+\frac{k_{1} k_{2}+k_{2} k_{0}+k_{1} k_{0}}{M\left(k_{1}+k_{0}\right)} y=\frac{k_{0}}{M\left(k_{1}+k_{0}\right)} F_{0}
$$

This can be changed to

$$
\ddot{y}+2 \xi \omega_{n} \dot{y}+\omega_{n}^{2} y=\frac{k_{0}^{2}}{k_{1} k_{2}+k_{2} k_{0}+k_{1} k_{0}} A \omega_{n}^{2} \cos (\omega t)
$$

This can be further altered to

$$
\left\{\begin{array}{l}
\xi=\frac{c}{2 M \omega_{n}} \\
\omega_{n}=\sqrt{\frac{k_{1} k_{2}+k_{0} k_{2}+k_{1} k_{0}}{M\left(k_{1}+k_{0}\right)}}
\end{array}\right\}
$$

Then, the system steady-state response

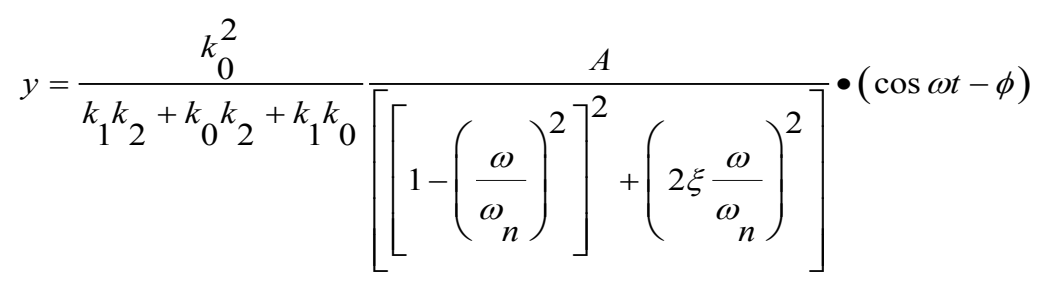

Here $\phi=\arctan \left[\frac{2 \xi \frac{\omega}{\omega_{n}}}{1-\left(\frac{\omega}{\omega_{n}}\right)^{2}}\right]$. 
The system displacement amplified time is

$$
|H(\omega)|=\frac{\frac{k_{0}^{2}}{k_{1} k_{2}+k_{0} k_{2}+k_{0} k_{1}}}{\left.\left[1-\left(\frac{\omega}{\omega_{n}}\right)^{2}\right]^{2}+\left(2 \xi \frac{\omega}{\omega_{n}}\right)^{2}\right]}
$$

When the excitation frequency is the system resonance frequency $\omega_{d}=\omega_{n} \sqrt{1-2 \xi^{2}}$, Equation (5) is

$$
k_{1}=\frac{k_{0}}{\frac{k_{0}}{M \omega_{d}^{2}}-1}
$$

From Equation (7),

$$
|H(\omega)|=\frac{\frac{k_{0}^{2}}{k_{1} k_{2}+k_{0} k_{2}+k_{0} k_{1}}}{\xi \sqrt{1-\xi^{2}}}
$$

To keep the material feeding efficiency, the feeder usually works under resonance conditions. While the loading increases, the mass $M$ increases accordingly. From Equation (5), $\omega_{n}$ reduces, and the system resonance frequency decreases accordingly. In Equation (8), when the mass $M$ increases, the stiffness $k_{1}$ increases accordingly, which enables the resonance frequency to be unstable. From Equation (7), when the displacement magnification factor $|H(\omega)|$ reduces, the feeding speed decreases; when the mass $M$ reduces, the stiffness $k_{1}$ decreases accordingly, and the system resonance frequency can stay stable without changes, but the displacement magnification factor $|H(\omega)|$ increases, and the feeding speed increases.

Figure 3. Dynamic model of the magnetic spring piezoelectric vibration feeder.

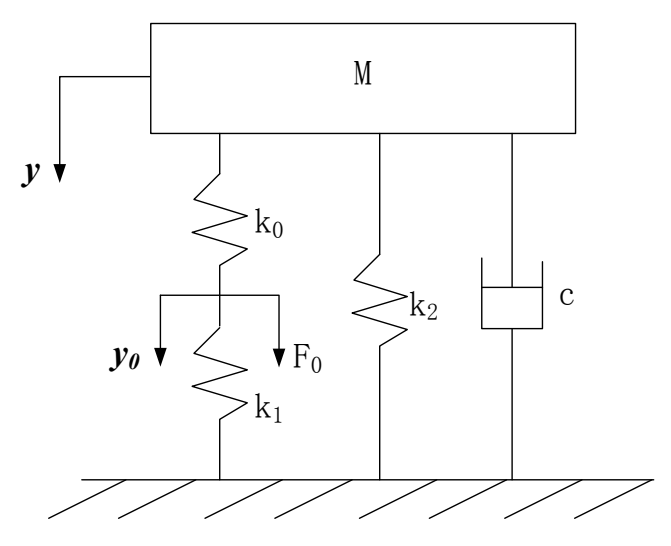

\section{Performance Experiment}

The excitation voltage is $200 \mathrm{~V}$, the detailed information please refer to Table 1 below: 
Table 1. Main components information.

\begin{tabular}{ccccc}
\hline Item & Material & Outer diameter $(\mathbf{m m})$ & Inner diameter $(\mathbf{m m})$ & Thickness $(\mathbf{m m})$ \\
\hline Annular ceramic plate* & $60 \mathrm{Si}_{2} \mathrm{MnA}$ & 50 & 10 & 0.6 \\
Annular base plate & $65 \mathrm{Mn}$ & 72 & 4 & 1.8 \\
Annular magnet & $\mathrm{NdFeB}$ & 60 & 15 & 10 \\
Top plate & Aluminum Alloy & \multicolumn{3}{c}{ Four springs located every $90^{\circ}$, tilt } \\
Supporting spring & $65 \mathrm{Mn}$ & \multicolumn{2}{c}{ angle $75^{\circ}$ between base and top plate } \\
Base & $45 \#$ & & \\
Vibration-reducing & Black rubber & & \\
spring & & & \\
\hline
\end{tabular}

*: especially made for the piezoelectric vibrator.

Figure 4 shows the experiment equipment. The equipment includes an Agilent HP4294 precise impedance analyzer, SDVC40-M digital piezoelectric frequency modulation controller, Keyence LC-2400 laser micrometer, electronic weighing device, tray, and materials for feeding. The prototype is shown in Figure 5.

Figure 4. Experiment equipment.

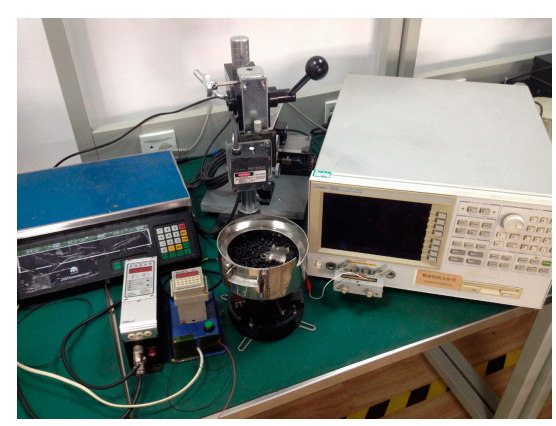

Figure 5. Prototype of piezoelectric vibration feeder with magnetic spring.

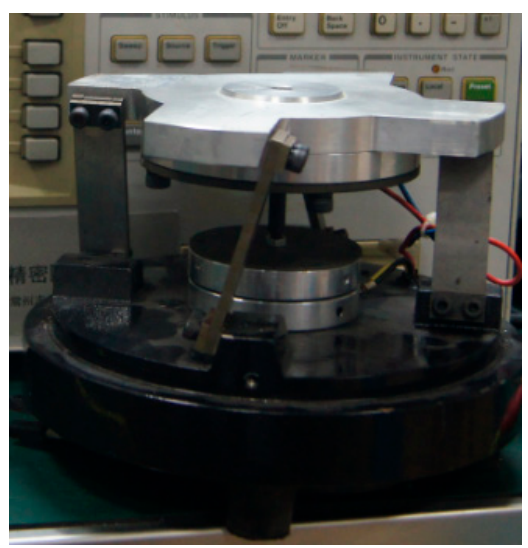

\subsection{Experiment on Loading and System Resonance Frequency}

The loading (material mass) and system resonance frequency of piezoelectric vibration feeder with magnetic spring relationship curve is shown in Figure 6, measured with the precise resistance analyzer while the feeder is working. 
Figure 6. Relationship between loading and resonance frequency.

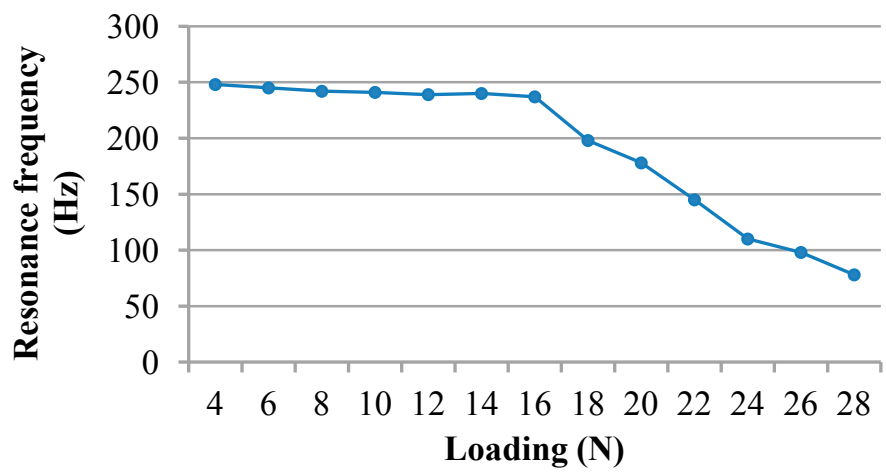

From the curve, as the loading is at $16 \mathrm{~N}$ and below, the prototype resonance frequency basically remains stable at $250 \mathrm{~Hz}$. If the loading is above $16 \mathrm{~N}$, the system resonance frequency dramatically reduces along with the increase of the loading. Therefore, the resonance frequency of the prototype is $250 \mathrm{~Hz}$, and the maximum loading is $16 \mathrm{~N}$.

\subsection{Experiment on the Relationship between System Loading and Vibration Displacement}

Set the driving frequency at $250 \mathrm{~Hz}$, loading increases gradually within $16 \mathrm{~N}$. The loading and vibration displacement relationship is shown in Figure 7.

In the figure, as the loading increases, the vibration displacement decreases slowly, which means the prototype feeding speed reduces slowly.

Figure 7. Relationship between system loading and vibration displacement.

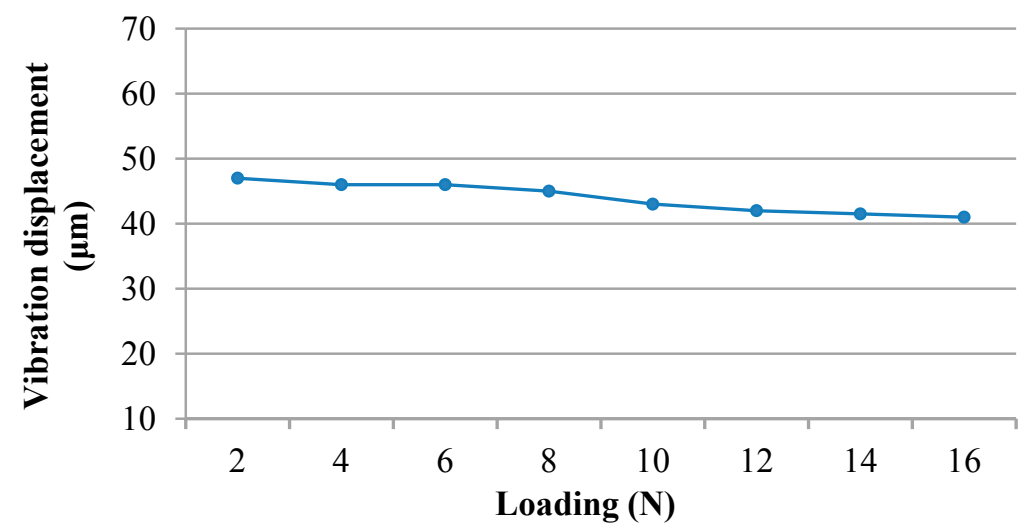

\subsection{Experiment on Relationship among Magnetic Spring Loading, Stiffness and Axial Clearance}

To validate the relationship among the magnetic spring stiffness, axial force and clearance, the magnetic spring axial force test is designed as is shown in Figure 8. The clearance, force and stiffness relationship is obtained, as is shown in Figures 9 and 10. The electronic weighing device and precise carving machine are glued together, and the bottom magnetic base is glued to the electronic weighing device. One annular magnet is placed on the bottom magnetic base; and another annular magnet is placed on the top magnetic base. Keeping the two annular magnets with the same polarity facing each other, the tail of top magnetic base is fixed with the clamp in the precise carving machine. In this 
setup, the precise carving machine is like a three freedoms of degree precise movement platform in $x, y$ and $z$ directions, and the controlling accuracy is $0.003 \mathrm{~mm}$. Moving the precise carving machine clamp in the $x$ and $y$ direction maintains the upper and lower magnets' concentricity. Moving the $z$ direction adjusts the axial clearance between the two magnets. The value from the electronic weighing device is the axial force between the two magnets.

Figure 8. The setup schematic diagram of magnetic spring experiment.

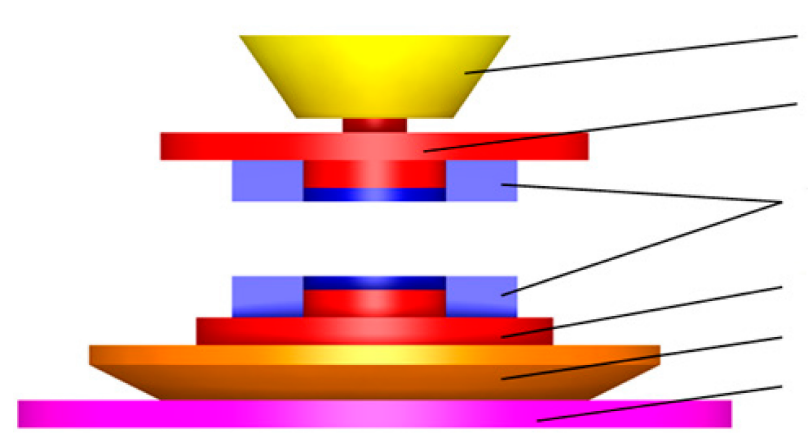

\section{Clamp}

Top magnetic base

\section{Annular magnets}

\section{Bottom magnet}

Electronic weighing device

Precise curving machine

Figure 9. Relationship between loading and axial clearance of the magnetic spring.

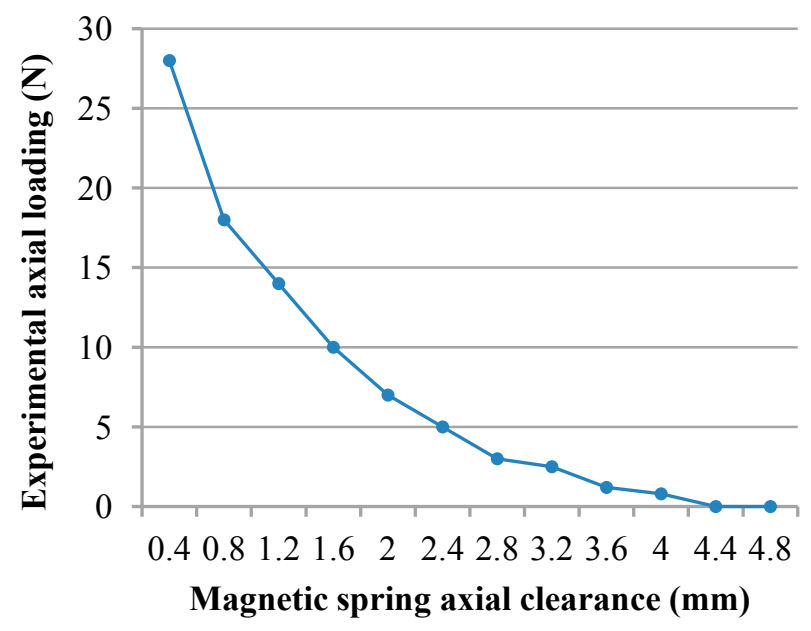

Figure 10. Relationship between stiffness and axial clearance of the magnetic spring.

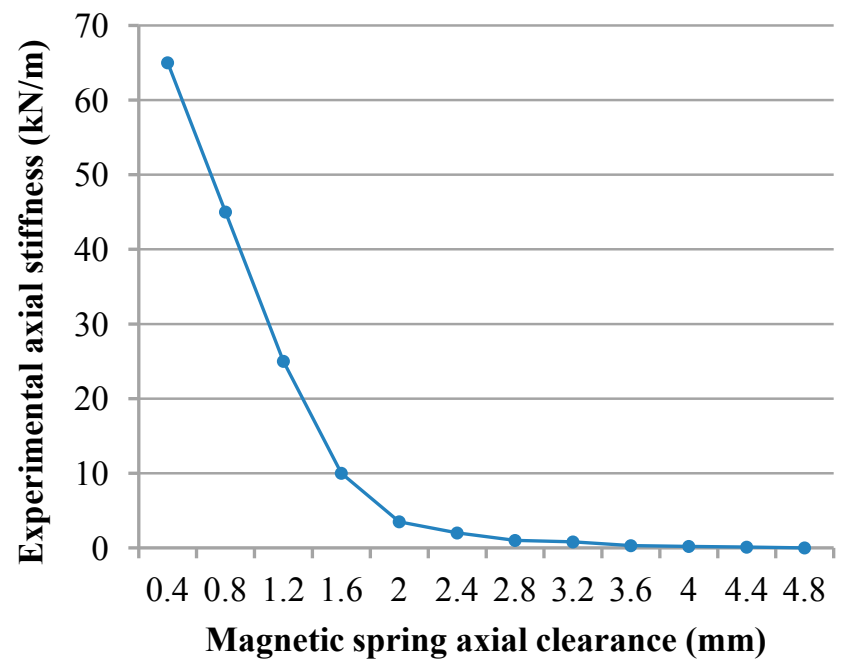




\subsection{Contrast Experiment}

We took the same size normal piezoelectric feeder, adjusted the signal generator, obtained voltage at $200 \mathrm{~V}$, turned the frequency into resonance frequency at the loading $16 \mathrm{~N}$ and put the materials on the trays separately. Meanwhile, we changed the materials mass; two different feeding speeds were measured, as is shown in Figure 11. From the figure, as the loading increases, the normal spring feeder speed decreases rapidly. However, the prototype feeding speed decreases gradually within the nominal loading capacity $(<16 \mathrm{~N})$, which basically keeps the material feeding speed stable.

Figure 11. Loading-speed curve on different spring feeders.

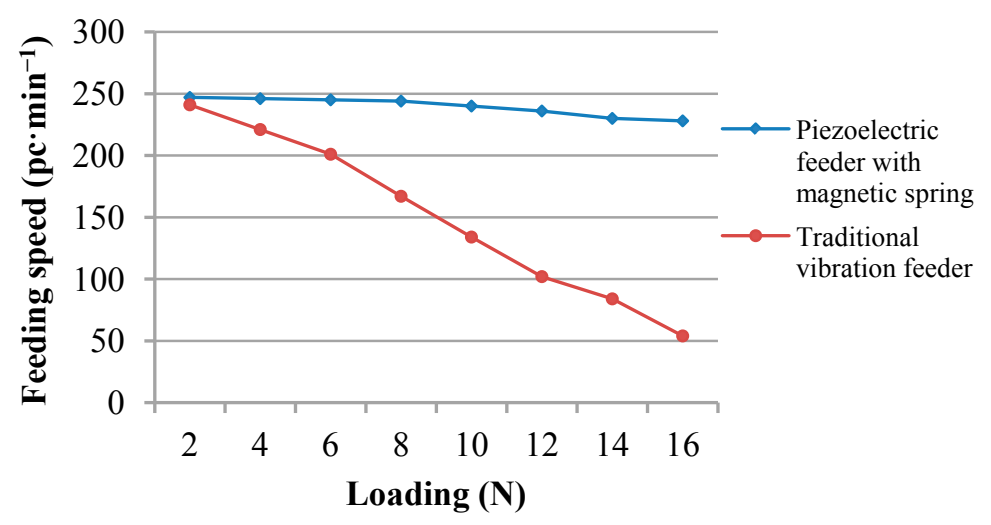

\section{Conclusions}

Based on the theoretical analysis in the paper, when the load of the piezoelectric vibration feeder with magnetic spring has synchronous changes with the magnetic spring stiffness, the system resonance frequency has the possibility to become stable. The prototype experiment on the piezoelectric vibration feeder with magnetic spring has validated that, when the material mass changes, the magnetic spring stiffness also changes due to the axial clearance changes in the magnetic spring. Therefore, the prototype resonance frequency and the feeding speed changes are not obvious in some loading range, which makes it more stable compared with traditional feeders.

\section{Acknowledgments}

The work is funded by the National Science Foundation of China (51075175), which is named "Structure Design Theory and Performance Experimental Research of Piezoelectric driven high frequency Fatigue Testing Machine".

\section{Author Contributions}

Yong Liu: responsible for the overall planning and task assignment, main composition and modification work in the paper. Xiaochao Tian: responsible for experiment, original data collection, composition of the paper draft. Zhigang Yang: instruct on the paper theory and experiment method. Yanhu Shen: responsible for deduction of the formula. Song Chen: responsible for figures and tables creation and prototype assembly. 


\section{Conflicts of Interest}

The authors declare no conflict of interest

\section{References}

1. Geoffrey, B.; translated by Xiong, Y.; Shan, C.; Lou, W. Assembly Automation and Product Design; China Machine Press: Beijing, China, 2009.

2. Huang, X. Structure improvement of electromagnetic vibrating feeder. Met. Mine Des. Constr. 2001, 33, 21-24. (In Chinese)

3. NTK Technical Ceramics. Piezoelectric Vibration Feeding Device. Japan Patent 52-61087, 4 May 1977.

4. Ruich, K.; Takara, F. Piezoelectric driven and piezoelectric element vibration feeder. China Patent 1380234A, 20 November 2002. (In Chinese)

5. Susumu, Y. Method and apparatus for controlling piezoelectric vibration. China Patent 1338668, 6 March 2002. (In Chinese)

6. Maul, G.P.; Thomas, M.B. A system model and simulation of the vibratory bowl feeder. J. Manuf. Syst. 1997, 16, 309-314.

7. Ting, Y.; Shin, M.S.; Chang, H.Y. Analysis and design of four-bar linkage type vibratory parts feeder driven by piezoelectric actuator. In Proceeding of ASME 2002 International Design Engineering Technical Conferences and Computers and Information in Engineering Conference, Montreal, Canada, 29 September-2 October 2002; Volume 2, pp. 43-50.

8. Choi, S.B.; Lee, D.H. Modal analysis and control of a bowl parts feeder activated by piezoelectric actuators. J. Sound Vib. 2004, 275, 452-458.

9. Chao, P.C.-P.; Chien, Y.-S. Dynamic modeling and experimental verification of a piezoelectric part feeder in a structure with parallel bimorph beams. Ultrasonics 2007, 46, 205-218.

10. Jiang, B.; Liu, X.L.; Yang, Z.G.; Tian, F.J.; Yu, Y. Study on vertical drive ultrasonic feeder. Opt. Precis. Eng. 2008, 16, 1082-1086. (In Chinese)

11. Zhang, G.L.; Guo, H.; Zhao, C.S. Ultrasonic powder-feeding device and its application. J. Vib. Meas. Diagn. 2001, 21, 186-189. (In Chinese)

12. Tian, Z.J.; Wu, W.F.; Han, F. The Experimental research on the floating piezoelectric vibratory feeder. Piezoelectrics Acoust. 2006, 28, 557-559. (In Chinese)

13. Jiao, Q.W.; Cui, W.H.; Sun, B.Y.; He, C.Y. Research and produce of piezoelectric vibration feeder. Transducer Technol. 2001, 20, 23-26. (In Chinese)

14. Tan, X.D.; Zhang, K. Mechanical analysis of the driven parts of piezoelectric vibration feeder. Manuf. Technol. Mach. Tool 2010, 3, 72-75. (In Chinese)

15. Su, J.; Yang, Z.G.; Zhang, C.J.; Shen, Y.H. Line vibratory feeder driven by circular piezoelectric vibrator. Trans. Chin. Soc. Agric. Mach. 2013, 44, 289-292. (In Chinese)

16. Li, Y.M. Research on a novel vibration bowel feeder and control system. Master's Thesis, Tianjin University, 25 May 2005.

17. Su, J.; Yang, Z.G.; Tian, F.G.; Shen, Y.H. Inertial piezoelectric vibratory feeder. Trans. Chin. Soc. Agric. Mach. 2013, 44, 281-286. (In Chinese) 
18. Qian, K.X.; Lu, L.C.; Ru, W.M.; Zheng, M.; Ma, L.Z. A magnetic spring and its elasticity. Chin. J. Mech. Eng. 1998, 34, 57-59. (In Chinese)

19. Cai, C.C.; Xu, Z.F.; Zhuang, J.P.; Yu, H. The analysis of working property for magnetic spring. Equip. Des. Maint. 2003, 6, 73-74. (In Chinese)

20. Yang, H.; Zhao, H. Study on dynamic characters of rare earth permanent magnetic spring. Trans. Chin. Soc. Agric. Mach. 2003, 34, 111-117. (In Chinese)

(C) 2014 by the authors; licensee MDPI, Basel, Switzerland. This article is an open access article distributed under the terms and conditions of the Creative Commons Attribution license (http://creativecommons.org/licenses/by/3.0/). 